\title{
The mental health and well-being benefits of exercise during the COVID-19 pandemic: a cross-sectional study of medical students and newly qualified doctors in the UK
}

\author{
Conor Coyle $^{1}\left(\mathbb{D} \cdot\right.$ Hanya Ghazi $^{2} \cdot$ loannis Georgiou $^{3}$ \\ Received: 20 October 2020 / Accepted: 21 October 2020 / Published online: 4 November 2020 \\ (C) The Author(s) 2020
}

\begin{abstract}
Background University students have been uniquely affected by the COVID-19 pandemic. However, there is currently little data upon the measures that medical students and newly qualified doctors have taken to help their mental well-being and mood during the COVID-19 pandemic.

Aim We aimed to identify the activities respondents found beneficial for their well-being and mental health and recorded a mood score from survey respondents.

Methods A nationwide study was completed to investigate the mental health impact of the COVID-19 pandemic upon medical students and newly qualified doctors (interim foundation year one). We received 2075 respondents from across the UK.

Results Physical activity was found to be the most common activity used by the survey respondents to help with their mental wellbeing $(80.1 \%$ ) (medical students, $83.7 \%$; interim foundation doctors, $72.3 \%$ ). Participants who stated that exercise helped their wellbeing had a mean score (SD) of $52.3(20.7)$ which was significantly higher $(P=0.048)$ than those who reported that they did not exercise (49.8 (21.1)). Respondents who stated they had used exercise to help with their mental well-being had (on average) a higher mood score than those who did not. This was seen in both the medical student and interim foundation doctor subgroups.

Discussion Exercise can help to benefit the well-being of medical students and interim foundation doctors. It is hoped that higher education providers and employers recognise the importance of promoting physical activity for the well-being of their students and staff, respectively.
\end{abstract}

Keywords Coronavirus · COVID-19 $\cdot$ Mental health $\cdot$ Medical students $\cdot$ Foundation doctors $\cdot$ Prospective study

\section{Introduction}

University students have been uniquely affected by the COVID-19 pandemic [1]. However, there is currently little data upon the measures that medical students and newly qualified doctors have taken to help their mental well-being and mood during the COVID-19 pandemic. As we adjust to the impact of COVID-19 upon our way of life, understanding actions that improve and support mental well-being become

Conor Coyle

M1501493@sgul.ac.uk

St. George's University of London, London, UK

2 Hull-York Medical School, York, UK

3 Aberdeen University, Aberdeen, UK especially important, in the development of future health policy and mental health support $[2,3]$.

Our group hypothesised exercise to be the most common activity of survey respondents. We also hypothesised that survey respondents who exercised would report higher mood scores.

\section{Methods}

A nationwide study was completed to investigate the mental health impact of the COVID-19 pandemic upon medical students and newly qualified doctors (interim foundation year one).

We received 2075 respondents from across the UK (aged 18-59); this included 1909 medical students (92.0\%) and 166 newly qualified doctors $(0.08 \%)$. We aimed to identify the activities respondents found beneficial for their well-being and mental health and recorded a mood score from survey respondents. Respondents were asked to provide a score from 
0 to 100 of their mood ( 0 being the worst and 100 being the best mood they could imagine). SPSS IBM v25 was utilised for the analysis of these results.

\section{Results}

The overall mean mood score of respondents was 51.8, SD 21.1. Physical activity was found to be the most common activity used by the survey respondents to help with their mental well-being $80.1 \%$ (medical students $83.7 \%$; interim foundation doctors $72.3 \%$ ). Participants who stated that exercise helped their wellbeing had a mean score (SD) of 52.3 (20.7) which was significantly higher $P=0.048$ than those who reported that they did not exercise, 49.8 (21.1). One-way ANOVA analysis revealed that there was a statistically significant difference $(P=0.037)$ between the mean mood scores (SD) of the following groups: students who did not exercise 49.7 (21.2), students who exercised 52.0 (21.0), doctors who exercised 56.2 (22.7) and doctors who did not exercise 50.9 (19.1).

Scheffe's post hoc analysis revealed that the statistically significant difference mentioned above was a result of the mean difference between students who did not exercise compared to doctors who exercised, who scored on average 6.5 points lower on mean mood scores.

\section{Discussion}

Respondents who stated they had used exercise to help with their mental well-being had (on average) a higher mood score than those who did not. This can be seen in both the medical student and interim foundation doctor subgroups. These results further demonstrate the benefits of physical activity upon well-being and provide a promising insight into the measures taken by medical students and interim foundation doctors in the UK, to help their mental well-being.

The promotion of exercise during the COVID-19 pandemic has become an objective of governments across the world. The health benefits of exercise and being physically fit have been widely supported as a measure to improve public health against the virus. Moreover, physical activity provides a means to manage negative emotions, positively impacting an individual's mental health and well-being $[4,5]$.

As COVID-19 continues to spread globally, medical students and interim foundation doctors will continue to face significant challenges and uncertainty surrounding their education, future careers and wider life. It is hoped that medical students and interim foundation doctors who do not exercise, engage in physical activity-recognising the benefits that physical activity can have for their health and well-being. It is also hoped that higher education providers and employers recognise the importance of promoting physical activity for the well-being of their students and staff, respectively.

Acknowledgments We would like to acknowledge the work of the additional members of the NANSIG collaborative - a group which the authors of this piece were a part of. Those we would like to acknowledge are as follows: Soham Bandyopadhyay, Marta de Andres Crespo, Ashok Handa, Conor S Gillespie, Bibire Baykeens, Mohammad Talha Bashir, Emily Bligh, Rohan Pancharatnam, Maria Georgiou, Shumail Mahmood, Anna Casey, Rosalind Di Traglia, Alex Fung, Jack Wellington, Adam Hounat, Jay J. Park, Joshua Erhabor, Mohammad H Ashraf, Lucas M Hernandez, Zeluleko Sibanda, Makinah Haq, Salma Mahmood, Abbey Boyle, Carlos M. Curtis-Lopez, Harry James Carr, Lorcán McMullan, Michael McLarnon, Armin Nazari, Emma Jane Norton, Guan Hui Tricia Lim, Oliver Rushworth and Kate E A Saunders.

\section{Compliance with ethical standards}

The authors of this paper declare that they have no conflicts of interest. All procedures performed involving human participants were in accordance with the ethical standards of the University of Oxford Medical Sciences Inter-Divisional Research Ethics Committee and with the 1964 Helsinki declaration and its later amendments. Informed consent was obtained from all individual participants included in the study.

Open Access This article is licensed under a Creative Commons Attribution 4.0 International License, which permits use, sharing, adaptation, distribution and reproduction in any medium or format, as long as you give appropriate credit to the original author(s) and the source, provide a link to the Creative Commons licence, and indicate if changes were made. The images or other third party material in this article are included in the article's Creative Commons licence, unless indicated otherwise in a credit line to the material. If material is not included in the article's Creative Commons licence and your intended use is not permitted by statutory regulation or exceeds the permitted use, you will need to obtain permission directly from the copyright holder. To view a copy of this licence, visit http://creativecommons.org/licenses/by/4.0/.

\section{References}

1. Zhang Y, Zhang H, Ma X, Di Q (2020) Mental health problems during the COVID-19 pandemics and the mitigation effects of exercise: a longitudinal study of college students in China. Int J Environ Res Public Health 17(10):3722

2. Matias T, Dominski FH, Marks DF (2020) Human needs in COVID19 isolation. J Health Psychol 25:871-882

3. Mandolesi L, Polverino A, Montuori S et al (2018) Effects of physical exercise on cognitive functioning and wellbeing: biological and psychological benefits. Front Psychol 9:509

4. Wang C, Pan R, Wan X et al (2020) Immediate psychological responses and associated factors during the initial stage of the 2019 coronavirus disease (COVID-19) epidemic among the general population in China. Int J Environ Res Public Health 17(5):1729

5. Brooks SK, Webster RK, Smith LE et al (2020) The psychological impact of quarantine and how to reduce it: rapid review of the evidence. Lancet 395:912-920

Publisher's note Springer Nature remains neutral with regard to jurisdictional claims in published maps and institutional affiliations. 